\title{
TÁCTICAS EN URETRA
}

\section{Marcelino G onzález Martín ${ }^{1}$, José Vicente Rodríguez y Carlos Rioja Sanz ${ }^{3}$}

${ }^{1}$ Servicio de Urología. Hospital Juan Canalejo. La Coruña

${ }^{2}$ Fundación Puigvert Barcelona. Barcelona.

${ }^{3}$ Servicio de Urología. Hospital Universitario M iguel Servet Zaragoza. España.

\section{ÍN DICE/ RESUMEN}

1. Esclerosis del cuello vesical.

M. González Martín.

2. Tácticas en uretrotomía ¿podríamos mejorar los resultados?.

J. Vicente Rodríguez.

3. Cuándo y qué prótesis.

C. Rioja Sanz.

Palabras dave: Tacticas en uretra. Esclerosis cuello vesical. Uretrotomía. Prótesis uretrales.

\section{ESCLEROSIS DE CUEUO VESICAL}

M. González Martín.

La esclerosis de cuello vesical aparece como complicación secundaria a la cirugía de próstata. Más frecuente en adenomectomía transvesical que en la retropúbica y más frecuente en la RTU, especialmente en próstatas pequeñas con componente inflamatorio (prostatitis, litiasis). (1). En una revisión de la Fundación Puigvert de 623 RTU de próstata, el 2,8\% desarrolla esclerosis de cuello vesical, con una media de 9 meses, especialmente en próstatas pequeñas (media 10,5 gramos). (2)

La esclerosis cierra progresivamente el cuello vesical formando un tabique que crece desde la celda prostática, dejando un pequeño orificio a las 12 del horario cistoscópico. La clínica de dificultad progresiva para orinar está en relación con el grado de obstrucción, llegando a desarrollar retención aguda de orina en el $30 \%$ de los casos (3)

Sea cual sea la causa de esta secuela debe ser resuelta mediante incisión, cervicotomía y no resección circular.

Con el resector, montando un asa de Collins, se realiza sección del tabique cervical a las 5 y 7 del horario cistoscópico, profundamente hasta llegar al tejido graso, desde la proximidad del meato ureteral hasta el veru montanum.

Dificultad especial surge cuando el tabique fibroso ha cerrado completamente la comunicación

\section{Marcelino G onzález M artín Servicio de Urología Hospital Juan Canalejo Xubias de Arriba, 84 15006 La C oruña (España)}


entre vejiga y uretra, entrando el paciente en retención por lo que precisa de talla suprapúbica.

\section{¿Cómo hacer la cervicotomía?}

1. Preparamos un catéter ureteral con aguja acoplada en su extremo.

2. A través de la sonda de talla llenamos vejiga mezclando con el suero, colorante de azul de metileno.

3. Pasamos por uretra, el uretrotomo con cuchillete de Sachse y el catéter ureteral con aguja.

4. Progresamos hasta el tabique cervical y perforamos éste con la aguja del catéter ureteral en la parte superior, a las 12 del horario cistoscópico.

5. Si obtenemos líquido azulado (orina teñida) por la luz del catéter, pasaremos paralelamente a la aguja el cuchillete del uretrotomo Sachse y realizamos una incisión en $Y$ invertida a las 5 y 7 del horario cistoscópico.

6. Retiramos el uretrotomo y pasamos el resector con asa de Collins para realizar a continuación la técnica antes descrita. (Figura 1)

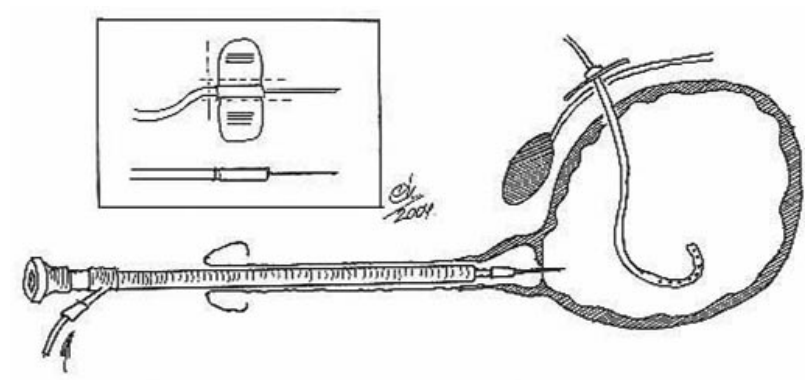

FIG URA 1. Esquema esclerosis de cuello. Esclerosis completa del cuello vesical. Esquema mostrando la maniobra de perforación del tabique con aguja montada sobre catéter ureteral. Detalle del montaje aguja (butterfly) sobre catéter.

\section{COMENTARIOS}

Se han empleado distintas técnicas para resolver el problema. Dilatación: $100 \%$ de recurrencia, RTU: $46 \%$ de recurrencia. Los resultados obtenidos mediante la cervicotomía oscilan entre 75 y $90 \%$ de éxitos. (4) Para intentar reducir el porcentaje de fracasos, de reesclerosis, se han utilizado inyecciones locales de corticoides u orgoteina (3). Es posible que sea beneficioso pero no está científicamente demostrado. La incisión con cuchillete frío mejora los resultados. (2)

El tratamiento con láser despertó fundadas esperanzas, ya que se produce inmediata vaporización y desintegración del área fibrosa con secundaria epitelización sin formar cicatriz. (5-6). En una o dos sesiones se alcanza el éxito prácticamente siempre.

\section{BIBUOGRAFÍA RECOMENDADA}

1. WEI, W.; GAO, J.; ZHANG, Z. y cols.: "Multifactor analysis of bladder neck contractures after transurethral resection of prostate". Zhonghua Nan Ke Xue, 10: 287, 2004.

2. HERRANDO, C.; BATISTA, J.E.; CHECHILE, G. y cols.: "Bladder neck sclerosis after transurethral resection of the prostate. Study Group of the Puigvert Foundation". Actas Urol. Esp. 18: 85, 1994.

3. GÓMEZ, F.; GONZÁLEZ, M.; SÁNCHEZ, J. y cols.: "Cervicotomia endoscópica en la esclerosis del cuello post-prostatectomía”. Arch. Esp. Urol., 44: 727, 1991.

4. SIKAFI, Z.; BUTLER, M.R.; LANE, V. y cols.: "Bladder neck contracture following prostatectomy". Br. J. Urol., 57: 308, 1985.

5. SILBER, N.; SERVADIO, C.: "Neodymium: YAG laser treatment of bladder neck contracture following prostatectomy". Lasers Surg. Med., 12: 370, 1992.

6. SALANT, R.L.; COHEN, M.S.; WARNER, R.S.: "Neodymium: YAG laser treatment of postoperative bladder neck contractures". Urology, 35: 385, 1990.

\section{TÁCTICAS EN URETROTOMÍA: ¿PODRÍAMOS MEJORAR LOS RESULTADOS?}

J. Vicente Rodríguez.

Según la bibliografía foránea y nacional, la uretrotomía endoscópica consigue buenos y estables resultados entre el 30-60\% de casos. Este porcentaje es inferior al conseguido con la uretroplástia (70$90 \%$ ), aunque sus buenos resultados también están condicionados a las características de la estenosis y al tiempo de seguimiento. Esta relevante diferencia debería estimularnos a conseguir mejorar los resultados de la uretrotomía sin alterar su simplicidad y 
reproductividad; para ello deberíamos seguir secuencialmente la siguiente táctica:

\section{Indicaciones precisas}

Para aumentar los buenos resultados 160 $75 \%)$, debemos reducir sus indicaciones. Debemos ser estrictos en la elección de casos tributarios de uretrotomía endoscópica: estenosis iatrógenas/ congénitas, menores de $2 \mathrm{~cm}$ de longitud, únicas (o dobles que sumadas no sobrepasen esta longitud), situadas en uretra bulbar y con poco espesor de espongiofibrosis.

Según nuestra experiencia (1), en un grupo de 100 pacientes, consecutivos, y sometidos a uretrotomía endoscópica: el $68,9 \%$ de la estenosis tenían características estrictas de indicación de cirugía endoscópica y en ella conseguimos aumentar el porcentaje de buenos resultados al 73,7\%, como muestra la Tabla I.

\section{Técnica depurada}

La técnica de la uretrotomía endoscópica es simple, sistematizada y conocida por abundante bibliografía urológica (1). Sin embargo su simplicidad no garantiza su correcta ejecución; al contrario relaja la exigencia técnica.

Por experiencia pretérita sabemos, que la incisión es preferible a la resección del "callo" fibroso; que la incisión a corte frío (cuchillete), es preferible al eléctrico (Collins) y que la segunda uretrotomía solo puede recaptar como buen resultado entre el 10-18\%, tras el fracaso de la primera (2). También conocemos por anterior / personal experiencia (Vicente. Eur. Urol. 1990), confirmado en la actualidad (Gurdal. J. Endourol 2003), que el láser N d YAg no aporta beneficio significativo a la incisión con cuchillete; semejantes resultados se han conseguido con láser argon y KTP (Malloy Urology 1992).

Creemos que la incisión a 12 horas siguiendo una técnica depurada y realizada con corte frío o con láser Holmium (3) consigue elevar los buenos resultados del 40-50 al 70\% ( (en casos de indicación cuidadosa de uretrotomía).

\section{Apoyo técnico}

La desobstrucción obtenida con la uretrotomía, debemos intentar mantenerla en el tiempo; conseguir estabilizar el calibre uretral, significa reducir la incidencia de estenosis y en definitiva aumentar el porcentaje de buenos resultados.

Para conseguirlos se han ensayado, como apoyo a la uretrotomía varios métodos con escasos resultados: autodilatación mecánica (con poca aceptación en nuestro medio), la dilatación intermitente con balón (Scott Urology 2000) y la inyección endoscópica con corticoides; su presunto éxito inicial (Hrader Eur. Urol. 7.1981), no se ha refrendado posteriormente ni tampoco en nuestra experiencia (1).

La autodilatación intermitente "limpia" con sondas ciegas autolubricadas y atraumáticas (tipo Lofric $\left.{ }^{\circledR}\right)$, ha demostrado en propia experiencia y en la bibliografía reducir francamente el índice de reestenosis, cuando se prolongan en el tiempo (Lawrence AUA 1991). Como muestra la Tabla II.

TABLA I.

\begin{tabular}{|l|lr|r|}
\hline CARACTERISTICAS & N N DE CASOS & $\%$ & BUEN OS RESULTADOS (\%) \\
\hline ETIO LO GÍA & IATRÓ GENAS & 52.6 & 57,9 \\
LO CALIZACIÓ N & BULBA R & 69.5 & 75,8 \\
LO N G ITUD & S1-2 CM & 66.3 & 74,6 \\
N ÚMERO & UN ICAS & 87.4 & 77,1 \\
\hline TOTAL & & 68.9 & $73,7 \%$ \\
\hline
\end{tabular}


TABLA II.

\begin{tabular}{|l|c|c|}
\hline \multicolumn{1}{|c|}{ Autor } & $\begin{array}{l}\text { Uretrotomía: } \\
\text { \% reestenosis }\end{array}$ & $\begin{array}{c}\text { Uretrotomía: } \\
\text { +autodilat \% }\end{array}$ \\
\hline $\begin{array}{l}\text { Kjaergaard } \\
\text { (Br.J.Urol.73.1994) } \\
\text { A lbers (3) } \\
\text { (J.Urol. 156.1996) } \\
\text { Harris } \\
\text { (Br.J.Urol.14.1994) }\end{array}$ & $68 \%$ & $19 \%$ \\
\hline
\end{tabular}

N osotros descartamos las prótesis uretrales auto / termoexpandibles como apoyo o ayuda a a umentar los buenos resultados de la uretrotomía ; sus indicaciones las limitamos a los casos de fracaso de uretrotonía repetidas 0 uretroplastias fallidas, como "última solución".

Con las prótesis se consigue excelente desobstrucción, pero no están exentas de efectos adversos, complicaciones y alto coste $(5,6,1)$.

\section{BIBUOGRAFÍA RECOMENDADA}

1. ROSALES, A.; VICENTE, J.: "Cirugía endoscópica del cuello y uretra". Tratado de endourología J. Vicente. Ed. Pulso, 6: 413, 1996.

2. HAYNS, C.F.; STEEMKAMP, J.W.; DE ROCK M.L. y cols.: "Treatment of male urethral stricture: is repeated dilation or internal urethrotomy useful?". J. Mol., 160: 356, 1998.

3. MATSONKA, K.; IMONE, M.; IRIDAS, S. y cols.: "Endoscopic anterograde laser incision in the treatment of urethral stricture". Urology, 968, 2002.

4. ALBERS, P.; FICHTNER, J.; BRÜHL, P. y cols.: "Long-term results of internal urethrotomy". J. Urol., 1611, 1996.

5. ASHKEN, M.H.; COULANGE, C.; MILROY, E.J.G. y cols.: "European experience with the utethral Wallstent for urethral strictures". Eur. Urol., 19: 181, 1991.

6. BALANI, G.; PRESS, S.; OOSTERLING, J. y cols.: "Urolume endourethral prothesis for the treatment stricture disesase: Long term results of North American Multicenter Urolume trial". Urology, 45: 846, 1995.

\section{CUANDO Y QUÉ PRÓTESIS URETRALES. \\ C. Rioja Sanz.}

Tenemos dos tipos de prótesis uretrales: definitivas y temporales. Las prótesis uretrales tuvieron una utilización amplia en la década de los 80 e inicios de los 90 . A ctualmente se han afianzado sus indicaciones, mucho mas limitadas.

Las prótesis permanentes han demostrado su eficacia en el tratamiento de las estenosis uretrales en los siguientes supuestos:

- Estenosis corta (1-2 cm.) recidivada

- Localización posterior

- Contraindicada en las estenosis traumáticas y uretra péndula

En general es una buena opción en las estenosis que han recidivado tras varios intentos de tratamiento endoscópico. Las estenosis largas son una mala indicación aunque en ciertos pacientes añosos, con mal estado general podría ser una indicación límite. En las estenosis de origen traumático el resultado es malo y muy frecuente el crecimiento de tejido intraprotésico.

Disponemos en el mercado de varios modelos aunque la mas extendida es la prótesis permanente URO LUME (Figura 1) que se presenta en el mercado en tamaños de $1.5-2-2.5-3-3.5-$ y $4 \mathrm{~cm}$. 0 tros tipos de prótesis, termoconfigurables o reabsorvibles (poliglicólico) prácticamente han desaparecido del mercado.

\section{Al colocarla debe tenerse en cuanta ciertos trucos:}

- Seccionar o dilatar la estenosis lo suficiente como para que la luz uretral tenga un calibre de 26 $28 \mathrm{Ch}$. que es el calibre que proporciona la prótesis. Si dilatamos menos, la prótesis se adaptará al calibre disponible y puede suponer problemas para endoscopias futuras

- Colocar tanto el extremo proximal como el distal en uretra sana y no lesionada, bien vascularizada. Para ello debemos medir correctamente el tamaño de la estenosis ya dilatada y colocar la prótesis a decuada, que sobrepase por lo menos $5 \mathrm{~mm}$. la zona estenótica. En caso contrario se producirá una nueva estenosis en los extremos protésicos. 
- Como el instrumento tiene la posibilidad de recolocar la prótesis antes de "soltarla" es conveniente estar muy seguros de su correcta ubicación.

- Evitar a toda costa que el extremo proximal quede alojado en uretra esfinteriana.

- No realizar maniobras endoscopicas a través de la prótesis por lo menos en 15 dias (incluido cateterismos uretrales) ya que movilizaríamos la prótesis. Si es necesario un drenaje vesical, colocar una talla suprapúbica.

\section{Manejo a medio y largo plazo de la prótesis y de las complicaciones}

Con una correcta indicación, las prótesis uretrales son una buena opción terapéutica y con escasas complicaciones. En el Hospital Universitario Miguel Servet con una serie de 87 prótesis uretrales colocadas, siguiendo estos criterios y más de 15 años de evolución, la tasa de buenos resultados es del $85 \%$.

- Si se produce una estenosis en los extremos (frecuente si la colocación no ha sido adecuada) el tratamiento consiste en solucionar la estenosis con el método elegido (uretrotomía o dilatación con balón) y colocar otra prótesis, generalmente corta $(1.5-2 \mathrm{~cm}$.) telescopada sobre la antigua y que cubra bien la nueva estenosis.

- Si se produce crecimiento de tejido intraprotésico (ocurre sobre todo en las estenosis traumáti-

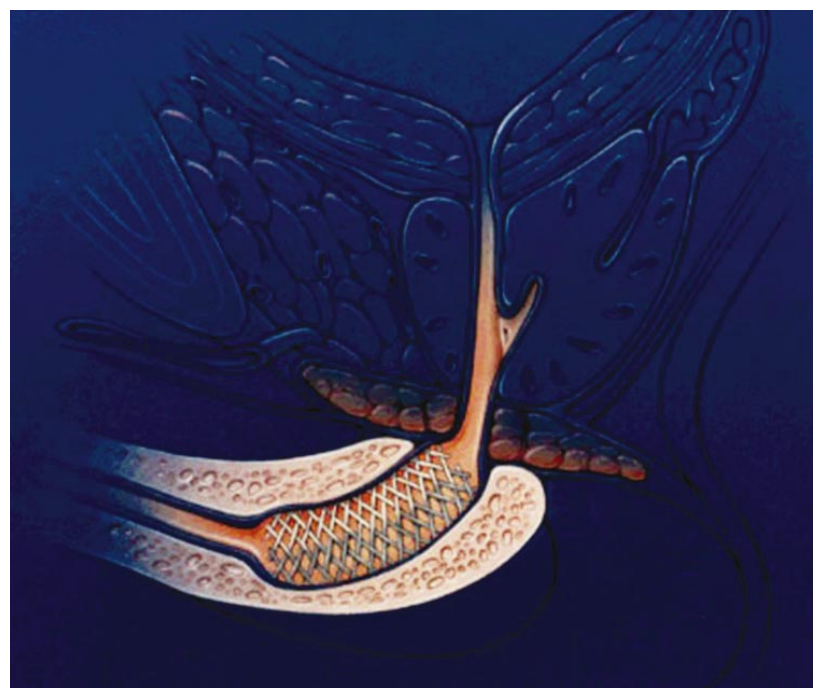

FIG URA 1. Prótesis autoexpandible uretral. cas) se puede retirar resecando el tejido con resección retrógrada. Esta técnica es eficaz pero con frecuencia difícil de realizar ya que un resector estándar de 24 $\mathrm{Ch}$ a veces cuesta pasarlo por la prótesis con el agravante de tener tejido intraluminal. Lo hemos resuelto usando resectoscopios finos, de los denominados "cadete" de $18020 \mathrm{Ch}$, disponibles en el mercado. 0 tro de los problemas es la transmisión de energía y calor a la uretra a través de los hilos metálicos de la prótesis que aparecen enseguida al resecar el tejido. A ctualmente hemos resuelto este problema de manera muy eficaz mediante la vaporización de este tejido con láser holmium. Se realiza con una fibra fina que pasa por cualquier panendoscopio a partir de $15 \mathrm{Ch}$.

- Como retirar la prótesis recién colocada: La retirada inmediata, antes de ser incluida tisularmente (primeros 15 días) se realiza con relativa facilidad anterogradamente con una pinza de extracción endoscópica. Si no fuera factible esta extracción existe el recurso de "empujar" la prótesis hasta la vejiga y

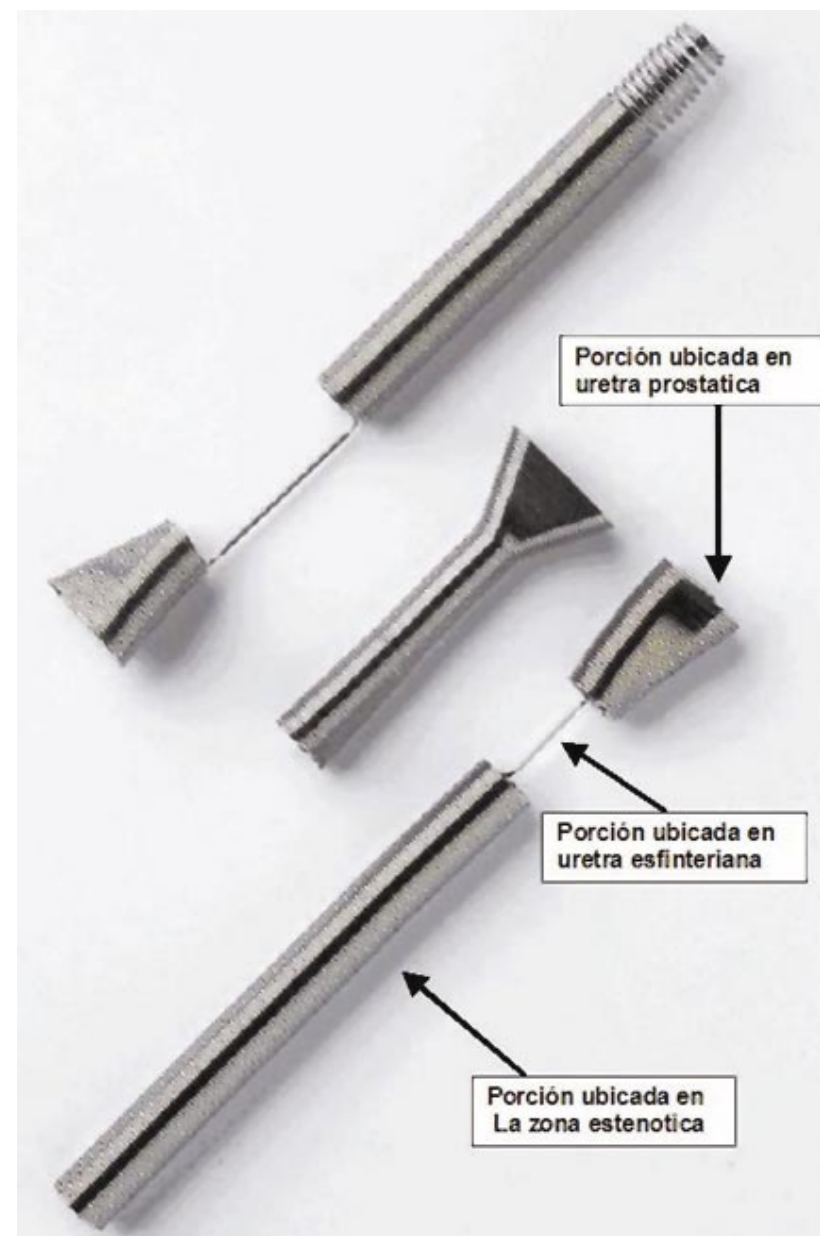

FIG URA 2. Prótesis uretral temporal. 
una vez allí extraerla por vía suprapúbica. En nuestra experiencia es muy útil la colocación de un trocar de laparoscopia por punción suprapúbica que se realiza bajo visión y control endoscopico. El trocar de $10 \mathrm{Mm}$. permite introducir un nefroscopio con pinzas potentes y extraer la prótesis por el propio trocar.

- Como retirar la prótesis integrada: A unque no tenemos experiencia pues no hemos tenido necesidad de ello, la literatura recoge la enorme dificultad que comporta retirar una prótesis totalmente integrada en el tejido. Generalmente este fenómeno se produce por malas indicaciones, por lo que insistimos en la importancia de que esta técnica debe ser rigurosamente indicada. Generalmente el motivo que justifica la retirada de prótesis es por crecimiento de tejido intraprotésico con imposibilidad de retirarlo o producción de un granuloma inflamatorio periprotésico con reestenosis de la uretra de forma distal y/ o proximal que requiere cirugía reparadora. Se ha descrito la retirada de los hilos por vía endoscópica, que debe de hacerse con una pinza y de uno en uno, circunstancia que parece compleja. Se ha descrito la necesidad de realizar una uretrectomía segmentaria que incluya la prótesis y todo el tejido dañado, realizando cirugía plástica reparadora en el mismo acto con o sin sustitución tisular.

Las prótesis temporales. Su objetivo sería sustituir durante un periodo corto de tiempo a una sonda uretral tras el tratamiento de una estenosis. De este modo se logra la tutorización de la uretra, con las ventajas de garantizar una cicatrización adecuada y manteniendo micción espontánea, sin catéter permanente. En el mercado disponemos de una prótesis uretral temporal (Figura 2) .Es una prótesis de una aleación metálica muy bien tolerada y que por su estructura no permite el crecimiento de tejido intraprotésico y por tanto su inclusión Su colocación es relativamente sencilla por vía endoscópica. Nuestra experiencia se basa en el tratamiento de estenosis muy complejas (traumáticas generalmente, con gran pérdida tisular y reparaciones complejas) en las que mantener una catéter largo tiempo nos permite evitar retracciones. La comodidad para el paciente es evidente, sin catéter permanente, se reduce el riesgo infectivo y logramos el objetivo de tutorizar la uretra periodos relativamente largos. Una porción de la prótesis queda alojada en uretra prostática, un fino filamento en zona esfinteriana y el resto, tutoriza la estenosis. 